\title{
Comparative Study on Receivers Performance Using DFE and LE Equalizer for Uwb Communication System
}

\author{
Shailja Rajput, Mr . Rakesh Mandal \\ M Tech Student Of Digital Communication Asst.Prof in shankaracharya bhilai C V R U Bilaspur,Kota
}

\begin{abstract}
In This paper comparison results are presented for receiver used for UWB communication system .we have taken in to account of impact of all the parameter such as Rake fingers and equalization tap on the error performance and SNR. Rake receivers can be employed since they are able to provide multipath diversity .another aspect is to combate the inter-symbol-interference(ISI), this distorts the transmitted signal. A semi analytical approach and mote-carlo simulation are used to investigate the BER performance of receivers on IEEE 802.15.3a UWB channel mode. we observe that the performance of MMSE Time domain equliser with DFE is high as compared to other receiver.
\end{abstract}

Index Terms: - UWB,BER,SNR,DFE,Arake, Prake, Srake receiver

\section{INTRODUCTION}

The trend of the modern wireless systems is to achieve higher data rates and better quality. The ultrawideband (UWB)communications is a possible technique to achieve this objective, due to its extremely large bandwidth. Ultra wideband(UWB) has recently evoked great interest and its potential strength lies in its use of extremely wide transmission bandwidth. Furthermore, UWB is emerging as a solution for the IEEE 802.15a (TG3a) standard which is to provide a low complexity, low cost, low power consumption and high data rate among Wireless Personal Area Network (WPAN) devices. An aspect of UWB transmission is to combat multipath propagation effects. Rake receivers can be employed since they are able to provide multipath diversity [1-3]. Another aspect is to eliminate or combat the inter-symbol interference (ISI)which distorts the transmitted signal and causes bit errors at , especially when the transmission data rate is very high as well as for which are not well synchronized.

In this paper we propose a simpler partial Rake (PRake)receiver structure, which combines the first arriving $L \mathrm{p}$ paths out of the available resolved MPCs.2 Thus this technique requires only synchronization, but not full channel estimation.3We compare the performance of PRake, SRake and ARake receivers that employ maximal-ratio combining [39]-[43] in realistic UWB channels.4 We analyze the signal-to-noise ratio(SNR) statistics at the combiner output and the bit error probability (BEP) of these Rake receivers using several widely used channel models. We consider both the IEEE 802.15.3achannel model, suitable for simulating UWB system that operate in the $3.1-10.6 \mathrm{GHz}$ range, and a channel model

that is based on baseband pulse measurements [29]-[31]. We analyze the influence of small-scale fading statistics and the influence of a "sparse" channel model (such as the IEEE(802.15.3a) on the performance of different Rake structures Finally, we investigate the dependence of both BEP and output SNR on the system bandwidth. We show that for a bandwidth of less than approximately $1 \mathrm{GHz}$, the performance improvement of the SRake as compared to the simpler PRake is quite small if the .fading is Nakagami (a typical case forUWB), whereas larger improvements result in Rayleigh fading

channels. For much larger bandwidths (up to $7.5 \mathrm{GHz}$ ), thePRake is not a good choice regardless of the fading statistics.

\section{CHANNEL MODELS}

We consider several UWB channel models to evaluate the receiver performance. A low-frequency (LF) model, proposed in [31] is based on experimental data collected in an office environment using baseband $1 \mathrm{~ns}$ pulses. The antennas further modified the pulse spectrum, especially at the lowest frequencies towards zero, resulting in a 3-dB bandwidth of about500 MHz, from 300-800 MHz. A high-frequency (HF) model,designed for 3.1-10.6 GHz, has recently been developed by the IEEE 802.15.3a for the simulation of FCC-compliant communication systems [32], [44].

The most important differences between this HF model and the LF model lie in the arrival statistics of the MPCs and in the distributions of MPCs' amplitudes. The HF model is sparse, i.e., there are resolvable delay bins that do not carry significant power mainly due to the use of much larger transmission bandwidths. Concerning the amplitudes' distributions, the HF model is lognormal while the LF model is Nakagami. The variance of the lognormal distribution of the HF model is assumed to be independent of delay, whereas the $m$ - 
parameters of the Nakagami distributions of the LF model decrease with delay. From the fig( 1) the performance of SRake and PRake in a channel with Rayleigh amplitude statistics. It can be seen that the performance of a PRake receiver is considerably worse than that of an SRake in such a channel, and the slopes ofthe CDFs are quite different. The reason for this is that for Rayleigh-fading taps

Summary of The Channel Models Used In The Analysis.

\begin{tabular}{|l|l|l|l|}
\hline & frequency & Amplitude & Comment \\
\hline LF model & $0.3-0.8 \mathrm{GHz}$ & $\begin{array}{l}\text { Nakagami ( m varies with } \\
\text { delay) }\end{array}$ & Almostcontinuous raysexponential decay \\
\hline HF model & $\begin{array}{l}3.1-10.6 \\
\mathrm{GHz}\end{array}$ & Lognormal & $\begin{array}{l}\text { Sparse channel:some resolvabledelays are(very } \\
\text { high resolution) }\end{array}$ \\
\hline FilteredHF model & $3.1-3.6 \mathrm{GHz}$ & Tend toRayleigh & $\begin{array}{l}\text { First path is not necessa rily the strongest one } \\
\text { generally, PDP is sparse }\end{array}$ \\
\hline Rayleigh & $0.3-0.8 \mathrm{GHz}$ & Nakagamim $=1$ & Comparison with conven tional wireless system \\
\hline
\end{tabular}

there is a larger probability that one of the first taps is in a deep fade. For Nakagami fading, 9 onthe other hand, the fading depth is much smaller, so a tap that carries large average energy also has a high probability of carrying high instantaneous energy. Conversely, from the comparison of Fig. 1(a) with Fig. 1(b), it emerges that with an SRake, a Rayleigh-fading channel actually gives a higher average energy than a Nakagami-fading channel.

\section{CHANNEL ESTIMATION AND ADAPTIVEMMSE RAKE}

\section{Equalizer Receiver}

The proposed receiver structure for DS-UWB system is as shown in Fig. 2. In this section, the proposed receiver reduces intense multi-path destruction and severe ISI by using a combined adaptive Rake and equalizer structure referred to as the adaptive MMSE Rake-equalizer receiver. Due to the large transmission bandwidth, the UWB channel is characterized by a long root-mean square delay spread and the Rake receiver cannot always overcome the resulting ISI [16]. We therefore study equalization for DS-UWB systems. The channel characteristics are first estimated using the LMS adaptive algorithm and initial value by the training sequence of preamble. The structure of the preambleis shown in Table 2. An adaptive receiver channel estimation for DS-UWB system is investigated, which can improve the performance of bit error rate.

\section{Channel estimation by using LMSadaptive algorithm}

Equation (4) can be written as

$$
r(t)=\sum_{\mathrm{n}=1}^{\infty} b n \cdot g(t-n T s)+u(t)
$$

where $g(t)=w(t) * h(t)$

We employ the LMS adaptive algorithm perform channel estimation, Thus adaptive weight adjustment with the optimum step-size parameter is
$(\mathrm{n}+1)$
(n)
(n)
(n)
$\mathrm{g}=$
$\mathrm{g}+\mathrm{e}$
. $\mathrm{rp} / 11 \mathrm{rp} 11$

Therefore, $\mathbf{g}$ can be estimated at the iterative process in matrix form as $\mathbf{g}=[\mathbf{g} p 1, \mathbf{g} p, 2 \mathrm{gpL}]$ which contains channel information (The amplitudes and delays are incorporated

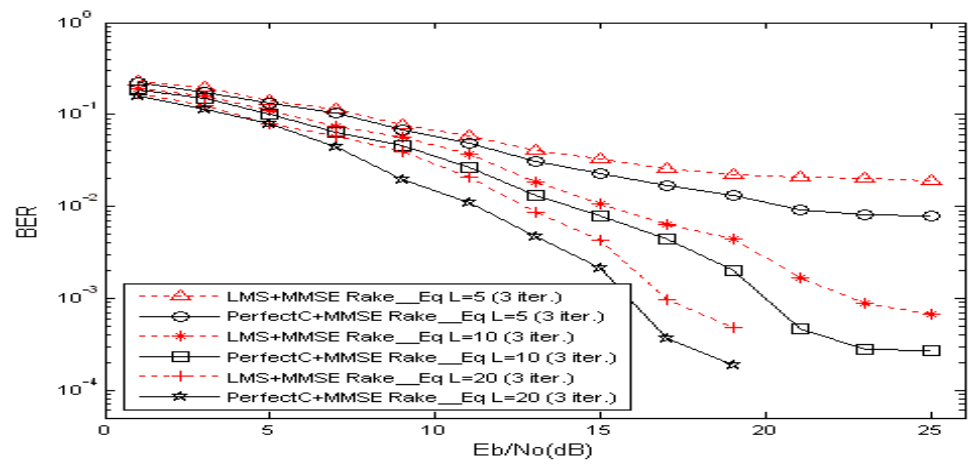

Fig-4-Bit error rate of an proposed receiver using LMS channel estimation and that of an proposed receiver with assumed perfect channel estimation for different numbers of Rake fingers for DS-UWB over CM4 channels 


\section{RAKE MMSE TIME DOMAIN EQUALIZER FOR HIGH DATA RATE}

For high data rate the proposed receiver combats inter symbol interference by taking advantage of the Rake and equalizer structure.BER performance observed on different UWB channel models (CM3 and CM4) shows that LE fails to perform satisfactorily at high SNR's due to presence of zeros outside the unit circle. These difficulty BER floor can be overcome DFE structure.

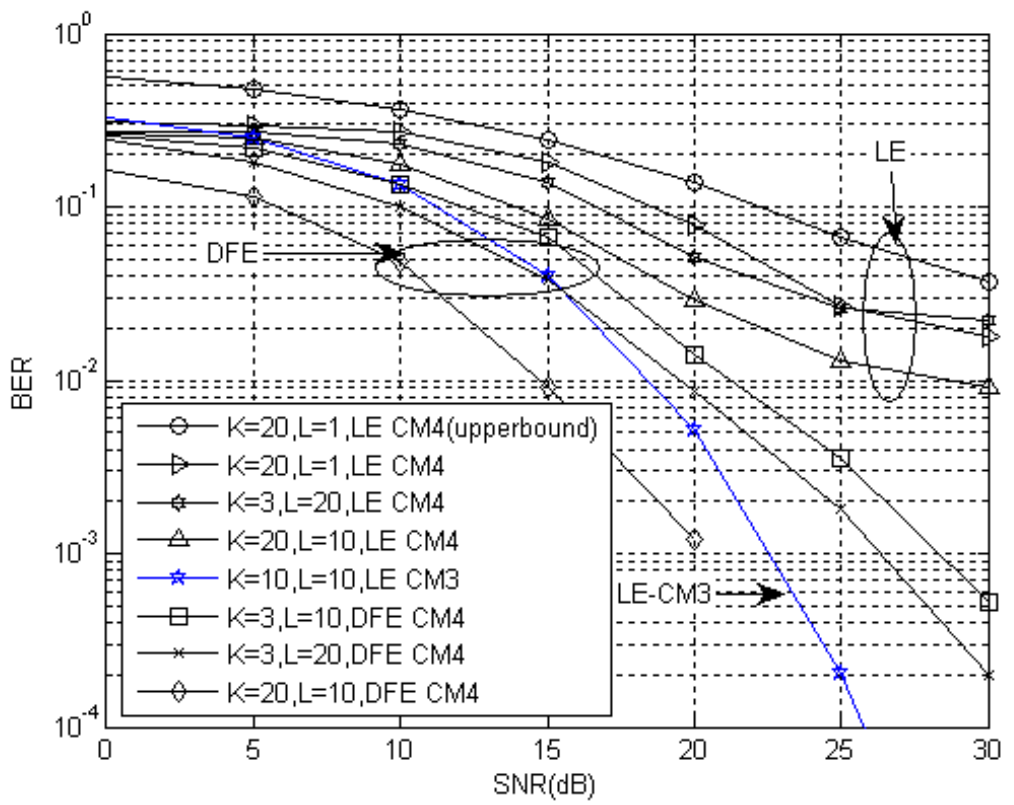

Figure.6. Performance of UWB S RAKE-MMSE receiver for different number of equalizer taps and RAKE fingers

\section{CONCLUSION}

We have analyzed the performance of Rake receivers in UWB indoor channels, we have concluded that the MMSE Rake equalizer needs LMS for cancelling channel distortions subsequent to obtaining the channel information via a training sequence. The proposed receiver is able to employ the energy of a few paths and obtain better performance by the addition of a channel estimation scheme . we have introduced a PRake architecture, which exploits only the first arriving propagation paths. PRake receivers are usually less complex than conventional Rake receivers, since they do not require a complete channel estimation or a full adaptability.We have compared the performance of the PRake with that of the more complex SRake in different channels. Due to the different behaviors of propagation media at different frequencies, the comparison based on channel models valid for frequencies either below $1 \mathrm{GHz}$ or above $3.1 \mathrm{GHz}$ has allowed us to highlight which particular Rake architecture ismost suitable for a given frequency range. we found that SRake receiver gives better performance ,because it select the path which has greater energy or SNR.

We found that the relative difference Adaptive rake, PRake and SRake strongly depends on the transmission bandwidth and the operating environment. The DFE equalizer gives better performance for UWB then linear equalizer .

\section{REFERENCES}

[1] A. Rajeswaran, V.S. Somayazulu and J. Foerster, "Rake performance for apulse based UWB system in a realistic UWB indoor channel", IEEE ICC'03., vol. 4, pp. 2879-2883, May 2003.

[2] S. Gezici, H. V. Poor and H. Kobayashi, "Optimal and Suboptimal Finger Selection Algorithms for MMSE Rake Receivers in Impulse Radio Ultra-Wideband Systems", in Proc IEEE WCNC 2005, NewOrleans, Mar. 2005.

[3] J. R. Foerster, "The effect of multipath interference on the performance of UWB systems in an indoor wireless channel", in Proc. 2001 Spring VehicularTechnology Conf., pp. 1176-1180, May 2001.

[4] J. D. Choi and W. E. Stark, "Performance of ultra-wideband communications with suboptimal receivers in multipath channels", IEEE J.Select. Areas Commun., vol. 20, pp. 1754-1766, Dec. 2002.

[5] D. Cassioli, M. Z. Win, F. vAtalaro and F. Molisch, "Performance of lowcomplexityRAKE reception in a Realistic UWB channel”, in Proc. Int. Conf.Commun. (ICC), vol. 2, May 2002, pp. 763-767. 
[6] T. Strohmer, M. Emani, J. Hansen, G. Papanicolaou and A. J. Paulraj,"Application of Time-Reversal with MMSE Equalizer to UWBCommunications", in Proc. IEEE Globecom'04., vol. 5, pp. 3123-3127, Dec. 2004.

[7] B. Mielczarek, M. O. Wessman and A. Svensson, "Performance ofCoherent UWB Rake Receivers With Channel Estimators", in Proc. VehicularTechnology Conf. (VTC), vol .3, Oct. 2003, pp. 1880-1884.

[8] A. Rajeswaran, V. S. Somayazulu and J. R. Forester, "Rake Performancefor a Pulse Based UWB System in a Realistic UWB Indoor Channel", in Proc.IEEE Int. Conf. Commun. (ICC), vol. 4, May 2003, pp. 2829-2833.

[9] Y. Ishyama and T. Ohtsuki, "Performance Evaluation of UWB- IR andDS-UWB with MMSE-frequency Domain Equalization (FDE)”, in Proc. IEEEGlobal Telecommun. Conf. (Globecom), vol. 5, Nov-dec 2004, pp. 3093-3097.

[10] J.F. Foerster, M. Pendergrass and A. F. Molisch, "A Channel Model for

[11] L. Yang and G. B. Giannakis, "Ultra-widebandcommunications: an idea whose time has come,"IEEE Signal Process. Mag., vol. 21, no. 6, pp.26-54, Nov. 2004.

[12] M. Z. Win and R. A. Scholtz, "Ultra-widebandwidth time-hopping spread-spectrumimpulse radio for wireless multiple-accesscommunications," IEEE Trans. on Comm., pp. 679-691, 2000.

[13] A. Rajeswaran, V. S. Somayazulu and J. R.Foerster, "Rake performance for a pulse based UWB system in a realistic UWB indoor channel," International Conference onCommunications, vol. 4, pp. 2879-2883, May 2003.

[14] J. Foerster and Q. Li, "UWB channel modeling contribution from Intel," IEEEP802.15-02/279-SG3a., Sept. 2002.

[15] Ning Xie and Yuanping Zhou, "An Adaptive Nonlinear Rake Receiver in UWB Wireless Communications," IEEE InternationalConference on Networking, Sensing and Control, pp. 480-485, 2325 April, 2006.

[16] H. Sheng, A. M. Haimovich, A. F. Molisch and J. Zhang, "Optimum Combining for Time Hopping Impulse Radio UWB Rake Receivers," IEEEConference on Ultra Wideband Systems and Technologies, pp. 224-228, Nov. 2003.

[17] Farahmand, S., Xiliang Luo and Giannakis, G.B., "Demodulation and tracking with dirty templates for UWB impulse radio: algorithms and performance," IEEE Transactions on VehicularTechnology, vol. 54, pp. 1595 - 1608, Sept. 2005.

[18] G.W. Rice, D. Garcia-Alis, I. G. Stirling, S. Weiss and R. W. Stewart, "An Adaptive MMSE Rake Receiver," Asilomar Conference on SSC, vol. 1, pp. 808-812, 29 Oct.-1 Nov. 2000.

[19] D. Falconer, S. L. Ariyavisitakul, A. B. Seeyarand B. Eidson, "Frequency domain equalizationsinglecarrier broadband wireless systems,"IEEE Commun. Mag., pp. 58-66, 2002 